\title{
Wave-induced steady streaming and net sediment transport in ocean bottom boundary layers
}

\author{
L. E. Holmedal \& D. Myrhaug \\ Department of Marine Technology, \\ The Norwegian University of Science and Technology, Norway
}

\begin{abstract}
The sediment transport resulting from the interaction between two important streaming-generating mechanisms has been investigated by numerical simulations of the seabed boundary layer beneath both sinusoidal waves and Stokes second order waves. These two mechanisms are streaming caused by turbulence asymmetry in successive wave half-cycles (beneath asymmetric forcing), and streaming caused by the presence of a vertical wave velocity within the seabed boundary layer.

Keywords: sea bed boundary layers, streaming, sediment transport.
\end{abstract}

\section{Introduction}

In coastal waters of intermediate or shallow water depths the surface waves induce water particle trajectories from the free surface to the bottom, dominating the flow in the water column. Near the bottom an oscillating boundary layer is formed because of the bottom friction. Inside this boundary layer, the wave-induced forcing is responsible for the transport of sea bed material either as bedload or as suspended load. This material includes sediments, chemical compounds, as well as biological material such as fish larvae.

Ocean surface waves are progressive, and for finite water depths the near-bottom water particle trajectories are ellipses where the horizontal axis is much larger than the vertical axis. Thus a small vertical wave velocity exists in the flow, and the existence of this vertical wave velocity gives rise to a weak mass transport within the oscillatory bottom boundary layer. This happens because the vorticity and turbu- 
lence created within the oscillating boundary layer are transported upwards from the bottom with time. Hence a weak vorticity exists in a layer much thicker than the oscillating boundary layer (Batchelor [3]). As a result, the vertical and horizontal velocity components are not 90 degrees out of phase within this layer (as they are in potential flow), and the vertical wave velocity combines with the horizontal wave velocity through the convective terms in the governing boundary layer equations, giving rise to a non-zero wave-averaged drift within the oscillatory boundary layer. This effect is caused by the bottom friction and wave action, and is commonly referred to as steady streaming. This streaming phenomenon for oscillating bottom boundary layers beneath gravity waves was first explained by LonguetHiggins [5]. However, steady streaming also arises because of wave asymmetry, as described in detail by Scandura [4] and Davies and Li [13] for flows in the transitional laminar to turbulent regime and flows in the rough turbulent regime, respectively. This phenomenon was first measured in an oscillating water tunnel by Ribberink and Al-Salem [1]. As pointed out by Scandura [4] the effect of wave asymmetry is particularly important in shallow waters. However, as explained by Longuet-Higgins [5], the steady streaming velocity will also be present in realistic near bottom flows beneath symmetric waves.

This work will focus on the sediment transport resulting from the interaction between two important streaming-generating mechanisms: The first is streaming caused by turbulence asymmetry in successive wave half-cycles (beneath asymmetric boundary layer forcing); the second is streaming caused by the presence of the vertical wave velocity within the seabed boundary layer as explained by Longuet-Higgins [5]. A more complete description is given by Holmedal and Myrhaug [12] including a detailed discussion of streaming-generating mechanisms and their physical implications.

\section{Model formulation}

\subsection{Governing equations}

Here the main governing equations and boundary conditions are given; for a more detailed description the reader is referred to Holmedal and Myrhaug [12]. Waveinduced mass transport in bottom boundary layers over an infinitely long flat bottom is considered. The horizontal coordinate at the bottom is given as $x$, whilst the vertical coordinate $z$ gives the distance from the bottom. The bottom is fixed at $z=z_{0}=k_{N} / 30$, where $k_{N}$ is the equivalent Nikuradse roughness. The limits of the horizontal coordinate $x$ is such that $x=0$ at the start of the wave length, and $x=\lambda$ at the end of the wave length. For intermediate and shallow water depths, the water particle trajectories are ellipses where the horizontal axis is much larger than the vertical axis. Hence the boundary layer approximation applies, and the simplified Reynolds-averaged equations for conservation of the mean momentum 
and mass become

$$
\begin{aligned}
\frac{\partial u}{\partial t}+\frac{\partial\left(u^{2}\right)}{\partial x}+\frac{\partial(u w)}{\partial z} & =-\frac{1}{\rho} \frac{\partial p}{\partial x}+\frac{\partial}{\partial z}\left(\nu_{T} \frac{\partial u}{\partial z}\right) \\
\frac{\partial u}{\partial x}+\frac{\partial w}{\partial z} & =0
\end{aligned}
$$

where $u$ is the horizontal velocity component, $w$ is the vertical velocity component, $p$ is the pressure, $\rho$ is the density of the water, and $\nu_{T}$ is the kinematic eddy viscosity. The turbulence closure is provided by a k- $\epsilon$ model. Subjected to the boundary layer approximation, these transport equations are given by (see e.g. Rodi [7])

$$
\begin{aligned}
& \frac{\partial k}{\partial t}+\frac{\partial(u k)}{\partial x}+\frac{\partial(w k)}{\partial z}=\frac{\partial}{\partial z}\left(\frac{\nu_{T}}{\sigma_{k}} \frac{\partial k}{\partial z}\right)+\nu_{T}\left(\frac{\partial u}{\partial z}\right)^{2}-\epsilon \\
& \frac{\partial \epsilon}{\partial t}+\frac{\partial(u \epsilon)}{\partial x}+\frac{\partial(w \epsilon)}{\partial z}=\frac{\partial}{\partial z}\left(\frac{\nu_{T}}{\sigma_{\epsilon}} \frac{\partial \epsilon}{\partial z}\right)+c_{\epsilon 1} \frac{\epsilon}{k} \nu_{T}\left(\frac{\partial u}{\partial z}\right)^{2}-c_{\epsilon 2} \frac{\epsilon^{2}}{k}
\end{aligned}
$$

where $k$ is the turbulent kinetic energy and $\epsilon$ is the turbulent dissipation rate. Here Eq.(2) has been applied to write Eqs.(1), (3) and (4) in conservative form. The kinematic eddy viscosity is given by

$$
\nu_{T}=c_{1} \frac{k^{2}}{\epsilon} .
$$

The standard values of the model constants have been adopted, i.e. $\left(c_{1}, c_{\epsilon 1}, c_{\epsilon 2}\right.$, $\left.\sigma_{k}, \sigma_{\epsilon}\right)=(0.09,1.44,1.92,1.00,1.30)$.

The instantaneous dimensionless bedload transport $\Phi$ is a function of the instantaneous dimensionless sea bed shear stress (Shields parameter) $\theta$ and is given by a formula by Nielsen [2]

$$
\Phi=12 \theta^{\frac{1}{2}}\left(\theta-\theta_{c}\right) \frac{\theta}{|\theta|} .
$$

where

$$
\begin{aligned}
\Phi & =\frac{q_{b}}{\left(g(s-1) d_{50}^{3}\right)^{\frac{1}{2}}}, \\
\theta & =\frac{\tau_{\mathrm{b}}}{\rho g(s-1) d_{50}} .
\end{aligned}
$$

Here $q_{b}$ is the instantaneous dimensional bedload transport, $\tau_{\mathrm{b}}$ is the dimensional instantaneous sea bed shear stress, $g$ is the gravity acceleration, $s=2.65$ is the density ratio between the bottom sediments and the water, $\rho$ is the water density, and $d_{50}$ is the median grain size diameter. The critical Shields parameter $\theta_{c}=0.05$ must be exceeded for bedload transport to take place. 
By using the boundary layer approximation, the equation for the sediment concentration $c$ is written

$$
\begin{aligned}
\frac{\partial c}{\partial t}+u \frac{\partial c}{\partial x}+\left(w-\mathrm{w}_{s}\right) \frac{\partial c}{\partial z} & =\frac{\partial}{\partial z}\left(\epsilon_{s} \frac{\partial c}{\partial z}\right), \\
\epsilon_{s} & =\nu_{T}+\nu .
\end{aligned}
$$

Here $\epsilon_{s}$ is the sediment diffusivity, $\mathrm{w}_{s}$ is the settling velocity of the median sand grains in still water, and $\nu$ is the laminar kinematic viscosity of water. Here the laminar viscosity has been included in the sediment diffusivity in order to stabilize the numerical scheme; this model is described in more detail in Holmedal et al. [6].

\subsection{Simplification of equations}

In order to simplify the mathematical solution of Eqs.(1)-(4) and (9) the relation $\partial / \partial x=-\left(1 / c_{p}\right) \partial / \partial t$ is applied. This is an approximation which is only valid for weakly decreasing waves (i.e. the wave height decay over a wave length due to the energy dissipation is small); this will be discussed further in conjunction with Eq.(14). This approximation leads to the two-dimensional boundary layer equations (i.e. Eqs.(1), (3), (4) and (9)) reducing to spatially one-dimensional equations. Physically this transformation implies a mapping from two spatial dimensions to one spatial dimension. The length of the physical two-dimensional space is one wave length, and the height is $z_{\max }-z_{0}$; in one dimension the height $z_{\max }-z_{0}$. The results obtained in one dimension can be mapped back to the physical twodimensional space.

As a consequence of this simplification the vertical velocity component is found from the continuity equation and is evaluated as

$$
w=-\int_{z=z_{0}}^{z} \frac{\partial u}{\partial x} d z=\frac{1}{c_{p}} \int_{z=z_{0}}^{z} \frac{\partial u}{\partial t} d z .
$$

and inserted into Eqs. (1), (3), (4) and (9). Here $c_{p}$ is the phase velocity which will be defined further below. Furthermore, $w=0$ at $z=z_{0}$ has been utilized (see Eq.(15)).

\subsection{Boundary conditions and numerical solution}

The sea bed is assumed to be hydraulically rough. At the bed ( $z=z_{0}$ ) no-slip conditions are imposed on the velocity. The $k-\epsilon$ model is coupled in a standard way with the logarithmic wall law near the bottom. Neumann conditions are imposed at the upper edge of the flow domain. This hydrostatic boundary layer flow is driven by progressive sinusoidal and second order Stokes waves. The boundary conditions for the sediment concentration are given by a specified reference concentration above the bed (depending on the Shields number) given by Zyserman and Fredsøe [8]. On top of the flow domain Neumann conditions are specified for the sediment concentration. 
A finite difference method was used to solve the governing equations, and the equations were integrated in time using the integrator VODE (Brown et al. [9]).

\section{Results and discussion}

This paper presents the wave-induced mass transport within the ocean bottom boundary layer for realistic wave conditions, bottom roughnesses, and water depth. Ocean surface waves with an amplitude of $a=1.22 \mathrm{~m}$ and a period of 6 s propagate over a flat rough bottom. The water depth is $8 \mathrm{~m}$, the resulting wave length is $45 \mathrm{~m}$, and the bottom roughnesses are, $z_{0}=1 \cdot 10^{-5}, 3 \cdot 10^{-5}, 6 \cdot 10^{-5}, 1 \cdot 10^{-4}$ and $2.3 \cdot 10^{-4} \mathrm{~m}$. By using the empirical formula $k_{\mathrm{N}}=2.5 d_{50}$, these roughnesses correspond to fine sand, medium sand, coarse sand, very coarse sand and gravel, respectively (Soulsby [10], Chapter 2). This wave condition represents intermediate water depth $\left(k_{p} h=1.11\right)$ with wave steepness $a k_{p}=0.17$. The near-bottom potential flow is approximated by second order Stokes theory.

\subsection{Streaming-induced sediment transport}

If the wave-induced forcing is strong enough to move the sea bed material (for example sediments and/or pollutants), or to bring it into suspension, then the weak streaming-induced boundary layer drift and non-zero wave-averaged bottom shear stress will cause a net transport of this material over time. This transport may take place either as net transport of suspended sediments or bedload.

Table 1: Mean bedload transport, suspended sediment transport and total sediment transport $\left(\bar{q}_{\text {total }}\right)$ for linear waves and Stokes second order waves. L.W denotes sinusoidal progressive waves; S.W denotes second order Stokes waves.

\begin{tabular}{|c|c|c|c|c|}
\hline & $d_{50}(\mathrm{~mm})$ & $\overline{q_{b}}\left(\mathrm{~mm}^{2} / \mathrm{s}\right)$ & $\int_{2 d_{50}}^{z_{\max }} \overline{u c} d z\left(\mathrm{~mm}^{2} / \mathrm{s}\right)$ & $\bar{q}_{\text {total }}\left(\mathrm{mm}^{2} / \mathrm{s}\right)$ \\
\hline L.W & 0.13 & 8.3 & 69.8 & 78.1 \\
\hline L.W. & 0.32 & 10.6 & 11.9 & 22.5 \\
\hline S.W & 0.13 & 10.2 & 57.9 & 68.1 \\
\hline S.W. & 0.32 & 13.3 & 12.4 & 25.7 \\
\hline
\end{tabular}

Table 1 shows the mean suspended sediment transport $\int_{2 d_{50}}^{z_{\max }} \overline{u c} d z$, the mean bedload transport $\overline{q_{b}}$ and the total sediment transport (the mean suspended sediment transport plus the mean bedload transport). It appears that the total sediment transport beneath second order Stokes waves is not very different from the total sediment transport beneath sinusoidal waves, given the uncertainty which is inherent in these sediment models. However, for $d_{50}=0.13 \mathrm{~mm}$ the total sediment 
transport is larger for sinusoidal waves than for second order Stokes waves, while for $d_{50}=0.32 \mathrm{~mm}$ the total sediment transport is larger for second order Stokes waves than for sinusoidal waves. In these simulations the settling velocities for the suspended sediments are $\mathrm{w}_{s}=0.0119 \mathrm{~m} / \mathrm{s}$ and $0.0429 \mathrm{~m} / \mathrm{s}$, for $d_{50}=0.13 \mathrm{~mm}$ and $d_{50}=0.32 \mathrm{~mm}$, respectively; these are taken from Dohmen-Janssen et al. [11].

Holmedal and Myrhaug [12] showed that both the Eulerian and the Lagrangian wave averaged seabed boundary layer velocity is larger beneath sinusoidal progressive waves than beneath Stokes second order waves. This has some implications for the resulting sediment transport. The trend seems to be that for fine sediments the suspended sediment flux is larger beneath sinusoidal waves than beneath second order Stokes waves, since fine sediments tend to follow the particle trajectories. Since the major part of the sediment transport is taking place as suspension for fine sediments, the total sediment transport is larger beneath sinusoidal progressive waves than beneath Stokes second order waves for fine sediments. For coarser sediments the trend is opposite: the total sediment transport is larger beneath Stokes second order waves than beneath sinusoidal progressive waves. For these coarser sediments the bedload yields a larger contribution to the sediment transport, and this contribution appears to be slightly larger for second order Stokes waves than for sinusoidal progressive waves. This might be due to that the maximum bottom shear stress during a wave cycle is larger for second order Stokes waves is larger than beneath sinusoidal progressive waves.

\section{Summary and conclusions}

The sediment transport resulting from the interaction between two important streaming-generating mechanisms has been investigated by numerical simulations of the seabed boundary layer beneath both sinusoidal waves and Stokes second order waves. These two mechanisms are streaming caused by turbulence asymmetry in successive wave half-cycles (beneath asymmetric forcing), and streaming caused by the presence of a vertical wave velocity within the seabed boundary layer.

It appears that the total sediment transport beneath second order Stokes waves is not very different from the total sediment transport beneath sinusoidal waves, given the uncertainty which is inherent in these sediment models. However, there is a trend showing that the total sediment transport is larger beneath sinusoidal progressive waves than beneath Stokes second order waves for fine sediments while as for coarser sediments this trend is opposite.

\section{References}

[1] Ribberink, J.S. \& Al-Salem, A.A., Sheet flow and suspension of sand in oscillatory boundary layers. Coastal Eng, 25, pp. 205-225, 1995. 
[2] Nielsen, P., Coastal bottom boundary layers and sediment transport. World Scientific Publishing Co. Pte. Ltd., Singapore, 1992.

[3] Batchelor, G.K., An Introduction to Fluid Dynamics. Cambridge University Press, 1967.

[4] Scandura, P., Steady streaming in a turbulent oscillating boundary layer. Journal of Fluid Mechanics, 571, pp. 265-280, 2007.

[5] Longuet-Higgins, M.S., Mass transport in water waves. Phil Trans $R$ Soc Lond A, 245, pp. 535-581, 1953.

[6] Holmedal, L.E., Myrhaug, D. \& Eidsvik, K.J., Sediment suspension under sheet flow conditions beneath random waves plus current. Cont Shelf Res, 24, pp. 2065-2091, 2004.

[7] Rodi, W., Turbulence Models and Their Application in Hydraulics, A stateof-the-art review. IAHR Monograph series, A. A. Balkema, Rotterdam, Netherlands, 3rd edition, 1993.

[8] Zyserman, J.A. \& Fredsøe, J., Data analysis of bed concentration of suspended sediment. J Hydr Res, 120(9), pp. 1021-1042, 1994.

[9] Brown, P.N., Byrne, G.D. \& Hindmarsh, A.C., VODE: A Variable Coefficient ODE Solver. SIAM J Sci Stat Comput, 10, pp. 1038-1051, 1989.

[10] Soulsby, R.L., Dynamics of marine sands. Thomas Telford Publications, 1997.

[11] Dohmen-Janssen, C.M., Hassan, W.N. \& Ribberink, J.S., Mobile-bed effects in oscillatory sheet flow. J Geophys Res, 106(C11), pp. 27103-27115, 2001.

[12] Holmedal, L.E. \& Myrhaug, D., Wave-induced steady streaming, mass transport and net sediment transport in rough turbulent ocean bottom boundary layers. Continental Shelf Research, 29, pp. 911-926, 2009.

[13] Davies, A.G. \& Li, Z., Modelling sediment transport beneath regular symmetrical and asymmetrical waves above a plane bed. Cont Shelf Res, 17(5), pp. 555-582, 1997. 